\title{
ON PLASTIC RELAXATION OF THERMAL STRESSES IN REINFORCED METALS
}

\author{
D. C. DUNAND and A. MORTENSEN \\ Department of Materials Science and Engineering, Massachusetts Institute of Technology, \\ Cambridge, MA 02139, U.S.A.
}

(Received 6 February 1990; in revised form 31 July 1990)

\begin{abstract}
Silver chloride containing alumina fibers or glass microspheres is used as a model material to study matrix plasticity induced by thermal mismatch in metal matrix composites. Resulting matrix dislocations are decorated at room temperature in the bulk material and observed by optical microscopy. Plastic deformation of the matrix around the inclusions is found to take the form of (i) rows of prismatic dislocation loops punched into the matrix and/or (ii) a plastic zone containing tangled dislocations surrounding the inclusions. From the number of loops punched by spheres, the temperature interval over which slip of prismatic loops is operative is calculated to be $100 \pm 30 \mathrm{~K}$ wide. The stress in the plastic zone around fibers is determined from the radius of curvature of pinned dislocations, leading to the conclusion that the matrix is locally strain-hardened. A simple model taking this fact into account is proposed to predict the radius of the plastic zone around embedded cylinders and spheres and is compared to the experimental data.
\end{abstract}

Résumé - Le chlorure d'argent contenant des fibres d'alumine ou des microsphères de verre est utilisé pour étudier la plasticité induite par incompatibilité thermique dans la matrice de matériaux composites à matrice métallique. Les dislocations qui en résultent sont décorées dans la matrice à température ambiante et observées par microscopie optique. La déformation plastique de la matrice autour de ces inclusions se manifeste par (i) des rangées de boucles de dislocations prismatiques et/ou (ii) une zone plastique contenant des dislocations emmélées autour des inclusions. A partir du nombre de boucles engendrées par les sphères, une valeur de $100 \pm 30 \mathrm{~K}$ est calculée pour l'intervale de température pendant lequel le glissement de boucles prismatiques est opérationel. La tension dans la zone plastique autour de la fibre est déterminée à partir du rayon de courbure de dislocations bloquées, ce qui conduit à la conclusion que la matrice est localement écrouie. Un modèle simple tenant compte de ce fait est proposé pour prédire le rayon de la zone platique autour de cylindres ou de sphères et comparé aux données expérimentales.

Zusammenfassung--Silberchlorid mit Aluminiumoxid Fasern oder Glassmikrokugeln wird als Modellmaterial benützt, um die thermisch induzierte Matrixplastizität in Verbundwerkstoffen mit metallischer Matrix zu studieren. Resultierende Matrixversetzungen werden bei Raumtemperatur im Materialinnern dekoriert und durch optische Mikroskopie beobachtet. Plastische Verformung der Matrix um die Einschlüsse wird in Form von (i) Reihen von in die Matrix ausgestossenen prismatischen Versetzungsringen und/oder (ii) einer plastischen Zone, die Versetzunganhäufungen um den Einschluss enthält, beobachtet. Aus der Zahl der von den Kugeln ausgestossenen Ringen wird das Temperaturinterval, während dessen Gleitung prismatischer Ringe wirksam ist, als $100 \pm 30 \mathrm{~K}$ ausgerechnet. Die Spannung in der plastischen Zone um die Fasern wird aus dem Krümmungsradius blockierter Versetzungen gefunden; aus diesem Resultat wird abgeleitet, dass die Matrix lokal verfestigt ist. Dementsprechend wird ein einfaches Model vorgeschlagen, das den Radius der plastischen Zone um Zylinder und Kugeln vorhersagt, und mit den experimentellen Daten verglichen.

\section{INTRODUCTION}

When the coefficients of thermal expansion (CTE) of the two phases of a composite are different, internal stresses are generated in the vicinity of the reinforcement upon temperature change. It is well documented that, if these thermal stresses are high enough, dislocations are produced in metal matrix composites (MMCs) near fibers, whiskers or particles. This phenomenon has been studied experimentally using etch pits [1, 2], slip lines [3] as well as transmission electron microscopy (TEM). This latter technique has revealed a variety of dislocation configurations surrounding the reinforcing phase, including long dislocations along fibers [4-6], dislocations emitted at whisker ends and corners $[7,8]$ as well as various loops and tangles $[9,10]$. There are shortcomings to these techniques, however, which impair complete observation of the actual dislocation configurations in reinforced metals. All these methods examine material in the immediate vicinity of a free surface, which perturbs both the dislocations and the stress state around the reinforcing phase. With TEM samples, furthermore, the observable volume in a given specimen is very small in comparison to even the smallest reinforcements of practical interest (whiskers), both in terms of thickness and of width of the electron-transparent area of observation. This 
restricts for example the observation of long dislocations or of entire rows of punched dislocations.

In the present investigation, we use silver chloride as a model material for the matrix of MMCs to alleviate these shortcomings. It is amply documented that this transparent salt exhibits substantial ductility (up to $400 \%$ tensile elongation [11]) resulting from dislocation mechanisms which are in most respects similar to those found in metals [12]. The glide direction of silver chloride is $\langle 110\rangle$ (corresponding to the most closely packed rows of ions of same sign) with glide planes $\{110\}$ and $\{001\}$ at ambient temperature, resulting in pencil glide. Single dislocations can be decorated at room temperature within the bulk of silver chloride or silver bromide by decoration with metallic silver, making them visible in high resolution transmission optical microscopy [13-16]. Decoration depths of $30 \mu \mathrm{m}$ can be reached by simple exposure to actinic light, a depth which can be extended to millimeters by the simultaneous use of a pulsed electrical field and pulsed light exposure [17-18]. Using plates of this material sufficiently thin for examination in the transmission optical microscope (up to dimensions on the order of millimeters), the whole sample can be observed. Consequently, decorated dislocations can be observed within undisturbed volumes as large as many square centimeters by over $30 \mu \mathrm{m}$, allowing the investigation of dislocations emitted along continuous fibers of large diameter or large particles. This technique has fallen out of fashion over the past decades and has its limitations (principally its low resolution), but the use of "transparent metals", as silver halides have been named, presents clear advantages over alternative techniques mentioned above for the study of matrix plastic deformation in MMCs.

In what follows, we present direct experimental evidence of dislocation emission around spherical and cylindrical reinforcing phases embedded in silver chloride. We then derive simple expressions predicting the size of the plastic zone that results in a strain-hardening matrix around these reinforcements, and compare our predictions with experimental data.

\section{EXPERIMENTAL}

Our experimental techniques closely follow ones established by Mitchell and co-workers [13-14, 19-23]. $99.999 \%$ pure silver chloride purchased from Engelhard was purified by melting under dry nitrogen and bubbling $99.9 \%$ pure chlorine, then degassed by bubbling dry nitrogen and finally filtered through a capillary to eliminate any particles, silver oxide or colloidal silver left in the melt. To increase its photosensitivity, the silver chloride was then doped with $500 \mathrm{ppm} 99.999 \%$ pure cuprous chloride, by melting both compounds under nitrogen and thoroughly mixing them in the melt. Throughout all operations involving the reactive molten silver chloride, great care was taken to exclude contami- nation by oxygen from air or by foreign ions from the Pyrex and quartz glassware used, which was therefore aged in boiling nitric acid and thoroughly rinsed in distilled water prior to each experiment.

Two types of reinforcements were used: alumina fibers with a diameter of $3 \mu \mathrm{m}$ (produced and donated by Imperial Chemical Industries, of trade name Safimax ${ }^{\mathrm{TM}}$ and composed of $95 \% \mathrm{Al}_{2} \mathrm{O}_{3}, 5 \%$ $\mathrm{SiO}_{2}$ ) and glass microspheres with a diameter range of 1-5 $\mu \mathrm{m}$ purchased from MoSci Corp. (borosilicate Corning glass 7070). The reinforcement was spread on a quartz plate on which $100 \mu \mathrm{m}$ thick quartz spacers were placed. Purified and doped silver chloride was melted under dry nitrogen and poured onto the quartz plate preheated to about $823 \mathrm{~K}$, which was then immediately topped by another hot quartz plate. The two plates were moved with respect to each other, thus creating turbulent flow within the melt which entrained the reinforcement from the surface of the plates to the bulk of the melt. The composite silver halide plate thus formed was directionally solidified at a speed of $10 \mu \mathrm{m} / \mathrm{s}$. After separation from the plates in distilled water, the composite was cut into smaller samples which were annealed in dry nitrogen for $2 \mathrm{~h}$ at $673 \mathrm{~K}$ and rapidly cooled down to room temperature at about $1 \mathrm{~K} / \mathrm{s}$. Each sample was then exposed for a few hours to the unfiltered light of a stroboscope (model 510AL, Electronic Brazing Company) with the following characteristics: Xe tube, 60 flashes/s, integrated illuminance per flash: $35 \mathrm{lux} / \mathrm{s}$. Immediately thereafter, the decorated substructure was observed in transmitted light and recorded photographically using an Olympus metallurgical microscope with a $100 \times$ dry lens and a short working distance condenser of aperture 0.65 .

\section{RESULTS}

Density differences between the melt and the reinforcement did not induce any separation of the two phases in the time needed for solidification. Even with the smallest glass spheres, no particle pushing by the solidification front was observed. In all cases, bubbles about a millimeter in diameter formed at the solidliquid interface during solidification, which could not be eliminated even when the sample was solidified vertically. They were probably due to shrinkage since the silver chloride was free of dissolved chlorine after the degassing operation. Being relatively few in number, they did not hinder the observation of the microstructure.

After annealing and quenching, all the sensitized samples showed small black surface precipitates which were due to the copper present in the samples, since non-sensitized control samples did not exhibit such a behavior. These precipitates were purely limited to the surface and did not seem to interfere at all with the microstructure in the bulk. Pulsed light of high intensity from the stroboscope was found to 


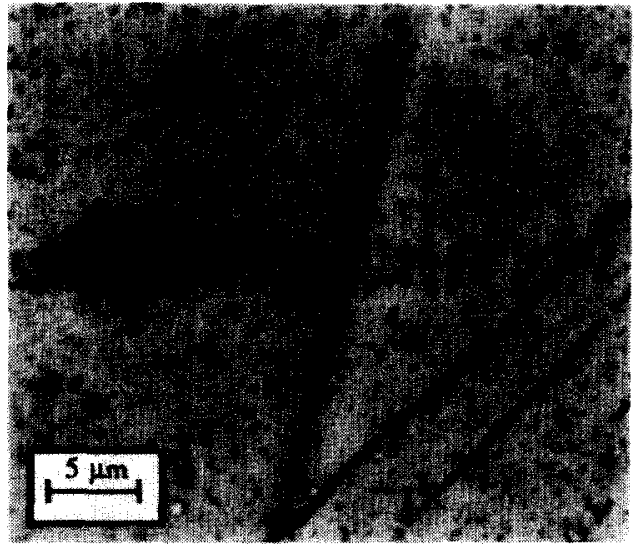

Fig. 1. Decorated prismatic loops in $\mathrm{AgCl}$ punched by a glass sphere. Unresolved tangles are visible in the directions where no loops were emitted.

yield better decoration of the samples than continuous exposure to the light of a mercury vapor lamp used by some previous investigators. In all samples, random precipitation of silver took place within the silver chloride upon exposure to actinic light, resulting in the "fog" visible in all figures. Grain boundaries and subgrain boundaries were always decorated first; when the samples were properly decorated and only slightly deformed, individual dislocations within the subgrains could be resolved. In most samples, decoration was found to fade in a matter of hours or days, more quickly so when the samples were exposed to the intense light of the microscope. Subsequent exposure to the stroboscopic light did not restore the decoration but rather increased the background fog.

\subsection{Samples with spheres}

High dislocations densities were observed in the vicinity of the glass spheres. The configuration of the resulting plastic zone surrounding the spheres fell in two main categories. In the first, the dislocations were clearly resolved as trains of prismatic loops emanating from the spheres along crystallographic directions. Unresolved tangles were almost always visible,

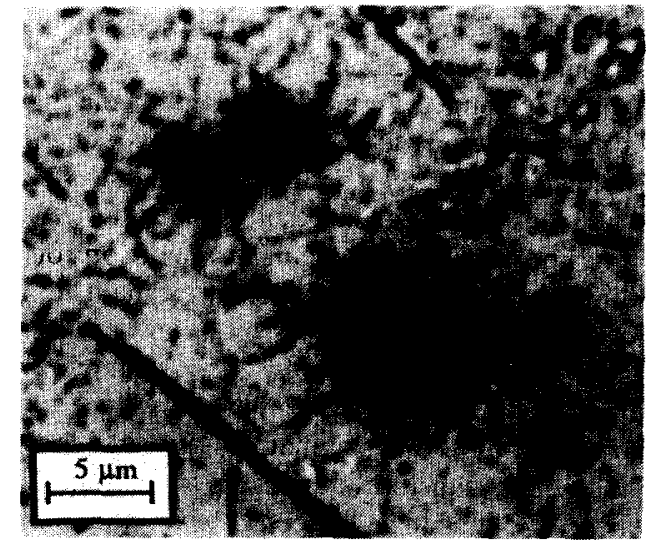

Fig. 2. Unresolved dislocations forming a spherical plastic zone around glass spheres embeded in $\mathrm{AgCl}$.

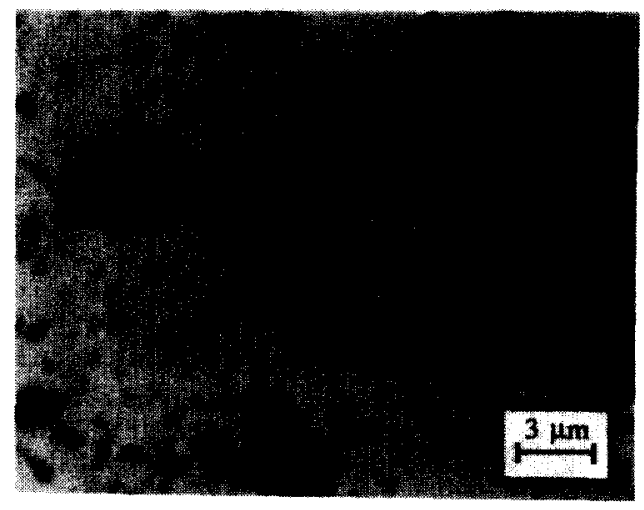

Fig. 3. Glass sphere in $\mathrm{AgCl}$ matrix surrounded by an irregular zone of unresolved, decorated dislocations and having emitted prismatic loops.

but only along the sides where no loops were punched (Fig. 1). In the other category, the plastic zone was composed of unresolved dislocation tangles surrounding the inclusion as a continuous shell of variable regularity (Fig. 2), on occasions coexisting with one or two trains of emitted loops (Fig. 3). In some cases of intermediate character in reference to these two types (Fig. 4), an irregular plastic zone was observed featuring lobes that extended along the glide directions (these were determined from other spheres having punched out loops within the same subgrain).

\subsection{Samples with fibers}

The dislocation density was found to be significantly higher close to the fibers than elsewhere in the matrix. Figure 5 shows dislocation tangles along the sides of a long fiber. Some of the dislocations have been pinned and are bowed due to the local stress field. In most cases, however, single dislocations could not be resolved within the plastic zone surrounding the fibers. The plastic zone could be seen as a dark cylindrical region of precipitated silver around the fiber. This region usually ended abruptly and was very often separated from the undeformed matrix by decorated subgrain boundaries.

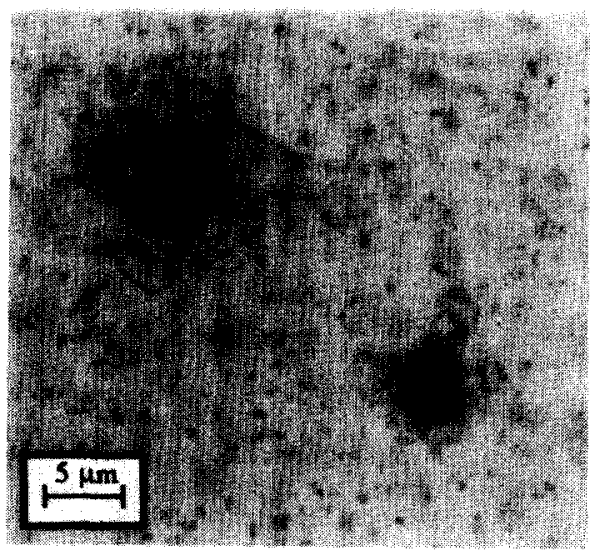

Fig. 4. Glass spheres in $\mathrm{AgCl}$ matrix surrounded by tangled, decorated dislocations in a plastic zone forming lobes. 


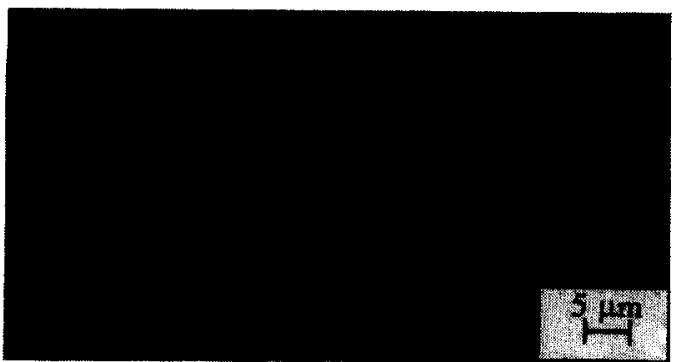

Fig. 5. Decorated, pinned dislocations in the plastic zone surrounding an alumina fiber in an $\mathrm{AgCl}$ matrix. Due to the stress from the fiber, some dislocations are bowed. The fiber is out of focus, being a few microns off the image plane.

In numerous cases, rows of prismatic loops were seen emanating from the ends of the fibers. In Fig. 6, a train of loops is shown which bursts through a subgrain boundary and continues in the next subgrain with a slightly different orientation, reflecting the change in crystallographic orientation between the two subgrains. Partially resolved tangles are also visible along the sides of the same fiber. Figure 7 shows a fiber surrounded by a dark plastic zone of unresolved dislocations. Many such cases were observed, where the plastic zone was larger at the ends of a fiber than as its middle. Due to the directional solidification of the samples, very large grains on the order of a $\mathrm{cm}^{2}$ were formed. Within these grains, subgrain boundaries - visible as dark continuous lines in Figs 1, 2,6 and 7-were always associated with regions of the sample containing fibers.

\section{THEORY}

The formation of a plastic zone around an inclusion embedded in metal when the misfit strain is

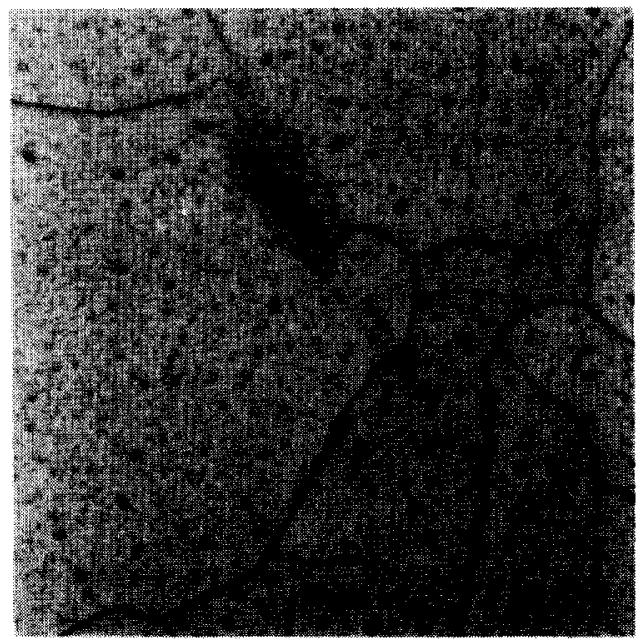

Fig. 6. Decorated rows of prismatic loops punched from a short alumina fiber in an $\mathrm{AgCl}$ matrix. After bursting through a subgrain boundary (dark continuous line), the loops change orientation in the second subgrain. Partially resolved dislocation tangles are also decorated around the fiber.

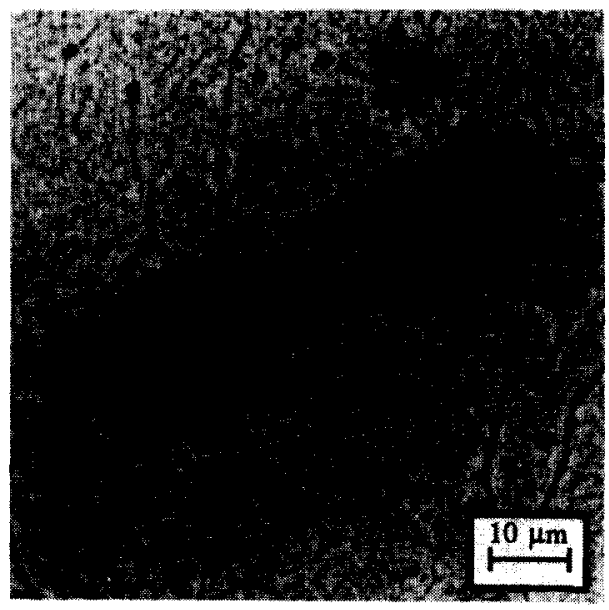

Fig. 7. Peanut-shaped plastic zone around an alumina fiber embedded in $\mathrm{AgCl}$.

pure dilatation has been modelled by various authors using a continuum approach $[3,8,24-32]$. In what follows, we propose an alternative method to calculate the size of the plastic zone surrounding inclusions of simple misfitting shape, namely spheres and cylinders embedded in a monocrystalline, elastically isotropic single-phase matrix. Our approach uses continuum plasticity as well, but takes into account the influence of dislocation density on flow stress.

Hill [32] solved the problem of the expansion of a cylindrical or spherical cavity into an infinite, isotropic, linear elastic, non work hardening plastic matrix from zero radius to end radius $a$. Assuming a Tresca yield criterion in the matrix, the radius of the plastic zone $c_{\mathrm{c}}$ and $c_{\mathrm{s}}$ for a cylinder and sphere of respective radius $a_{\mathrm{c}}$ and $a_{\mathrm{s}}$ are respectively given as

$$
\begin{aligned}
& c_{\mathrm{c}}=a_{\mathrm{c}} \cdot\left(\frac{2 \cdot E}{(5-4 v) \cdot \sigma_{\mathrm{y}}}\right)^{1 / 2}, \\
& c_{\mathrm{s}}=a_{\mathrm{s}} \cdot\left(\frac{E}{3 \cdot(1-v) \cdot \sigma_{\mathrm{y}}}\right)^{1 / 3}
\end{aligned}
$$

where $E$ is the matrix elastic modulus, $v$ the matrix Poissons ratio and $\sigma_{y}$ the matrix yield tensile stress.

These results can be used to find the plastic zone radius due to the differential contraction of an infinite matrix around an inclusion. Consider a sphere or an infinitely long cylinder embedded in an infinite matrix which deforms plastically. Upon cooling from elevated temperature, the matrix is first able to alleviate misfit stresses that result from the differential thermal contraction of the reinforcement by diffusion or other creep mechanisms. These mechanisms will operate as long as their rate is on a par with that of cooling. At low temperatures, creep cannot relieve thermal stresses, which build up and induce plastic deformation by slip in the matrix that surrounds the inclusion. The transition from creep to slip is assumed to take place at a single temperature $T_{\mathrm{c}}$, above which all thermal mismatch strains are relieved by diffusion, and below which dislocations form at the interface 


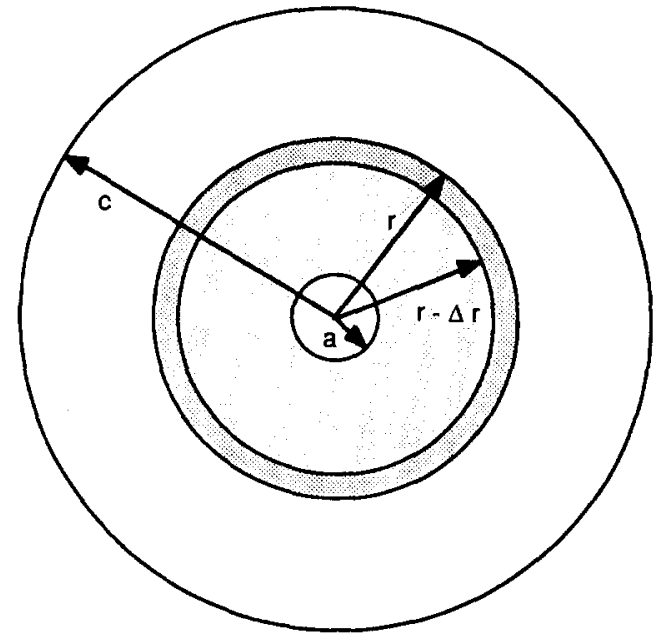

Fig. 8. Fictitious hole expanded from zero radius to radius $a$ producing a plastic zone of radius $c$ and a matrix displacement of $\Delta r$ at the surface of the inclusion of radius $r$. Cylindrical or spherical case.

and glide within the matrix to relieve thermal mismatch stresses higher than the yield stress of the matrix. Thus, upon cooling from a high annealing temperature $T_{\mathrm{a}}$ to a lower temperature $T_{0}$, stresses will be relieved first by diffusion and/or creep at high temperatures until temperature $T_{\mathrm{c}}$ is reached. At this temperature, the inclusion and the matrix are assumed to be stress-free. Upon further cooling to $T_{0}$, elastic stresses build up in both phases until the matrix yield stress is reached and slip begins in the matrix. It is assumed that the stress for nucleation of dislocations at the interface is not limiting the number of dislocations, as reported by Brown and Woolhouse [33] for two-phase materials with incoherent interfaces. Given the relatively low yield stress of silver halides (and most metals) and the high modulus of the inclusions of interest, the latter is assumed to be perfectly rigid. The total mismatch strain $\epsilon_{\mathrm{m}}$ between matrix and inclusion to be relieved by slip is

$$
\epsilon_{\mathrm{m}}=\Delta \alpha \Delta T
$$

where

$$
\Delta T=T_{\mathrm{c}}-T_{0}
$$

and $\Delta \alpha$ is the absolute value of the difference of CTE between matrix and inclusion. The CTEs are assumed to be isotropic and independent of the temperature. The displacement $\Delta r$ of the matrix due to the presence of an inclusion of radius $r$ at its surface is then known, and given as

$$
\Delta r=\Delta \alpha \cdot \Delta T \cdot r .
$$

By using equation (1), the radius of a fictitious cylindrical or spherical hole of radius $a_{\mathrm{c}}$ and $a_{\mathrm{s}}$, respectively, which would produce in an unreinforced matrix a displacement $\Delta r$ at the inclusion surface can be calculated (Fig. 8). Since the stress and strain state, as well as strain history of the matrix are identical for these two cases of a thermally mismatching inclusion and a growing hole, the size of the plastic zone can be found by deriving $a_{\mathrm{c}}$ or $a_{\mathrm{s}}$, and using equation (1). The radii $a_{\mathrm{c}}$ and $a_{\mathrm{s}}$ are most easily derived from conservation of volume, neglecting the elastic compressive strain within the inclusion volume in the growing hole configuration. Neglecting higher orders of $\Delta r$, one obtains for the cylinder and the sphere, respectively

$$
\begin{aligned}
\pi \cdot a_{\mathrm{c}}^{2} & =2 \cdot \pi \cdot r_{\mathrm{c}} \cdot \Delta r, \\
\frac{4}{3} \cdot \pi \cdot a_{\mathrm{s}}^{3} & =4 \cdot \pi \cdot r_{\mathrm{s}}^{2} \cdot \Delta r .
\end{aligned}
$$

Inserting equations (5a) and (5b) into equations (1a) and (1b) respectively and taking equation (4) into account, the radius of the plastic zone produced by differential thermal contraction of the matrix around a cylinder $\left(c_{c}\right)$ and a sphere $\left(c_{s}\right)$ is respectively given by

$$
\begin{aligned}
& c_{\mathrm{c}}=r_{\mathrm{c}} \cdot\left(\frac{4 \cdot \Delta \alpha \cdot \Delta T \cdot E}{(5-4 v) \cdot \sigma_{\mathrm{y}}}\right)^{1 / 2}, \\
& c_{\mathrm{s}}=r_{\mathrm{s}} \cdot\left(\frac{\Delta \alpha \cdot \Delta T \cdot E}{(1-v) \cdot \sigma_{\mathrm{y}}}\right)^{1 / 3}
\end{aligned}
$$

assuming that $E, v$ and $\sigma_{\mathrm{y}}$ are isotropic and temperature independent.

Equations (6a) and (6b) predict the extent of the plastic zone in a matrix showing no strain-hardening. From data presented below and measurements on pure silver chloride [34-36], however, it is known that this material exhibits strain-hardening, as most metals do. Use of macroscopic relations giving flow stress as a function of strain history is inappropriate given the microscopic dimensions of plastic flow. We assume that the matrix is monocrystalline, elastically isotropic and single-phased, and make use of the more "microscopic" relationship between dislocation density $\rho$ and flow stress of the matrix $\tau_{y}$ given by the classical equation [37]

$$
\tau_{\mathrm{y}}=\tau_{\mathrm{y} 0}+A G b \sqrt{\rho}
$$

where $\tau_{\mathrm{y} 0}$ is the intrinsic flow stress of the matrix, $G$ its shear modulus, $b$ the Burgers vector of dislocations in the matrix and the strain-hardening constant $A$ has a value between 0.3 and 0.6 for metals [38-43] and 0.4 for $\mathrm{NaCl}$ [44]. We assume for the case of interest here - namely the formation of a cylindrical or spherical plastic zone of tangled dislocations surrounding the inclusion - that the impeding effect of one dislocation upon motion of another can be represented by equation (7). The same relationship was used by Brown and Stobbs [45] to model strainhardening in the plastic zone around submicroscopic particles in a deformed copper-silica system. We make the added assumptions that flow stress and dislocation density are uniform within the plastic zone and that the plastic zone size always increases with increasing $\Delta T$. To calculate the extent of the 

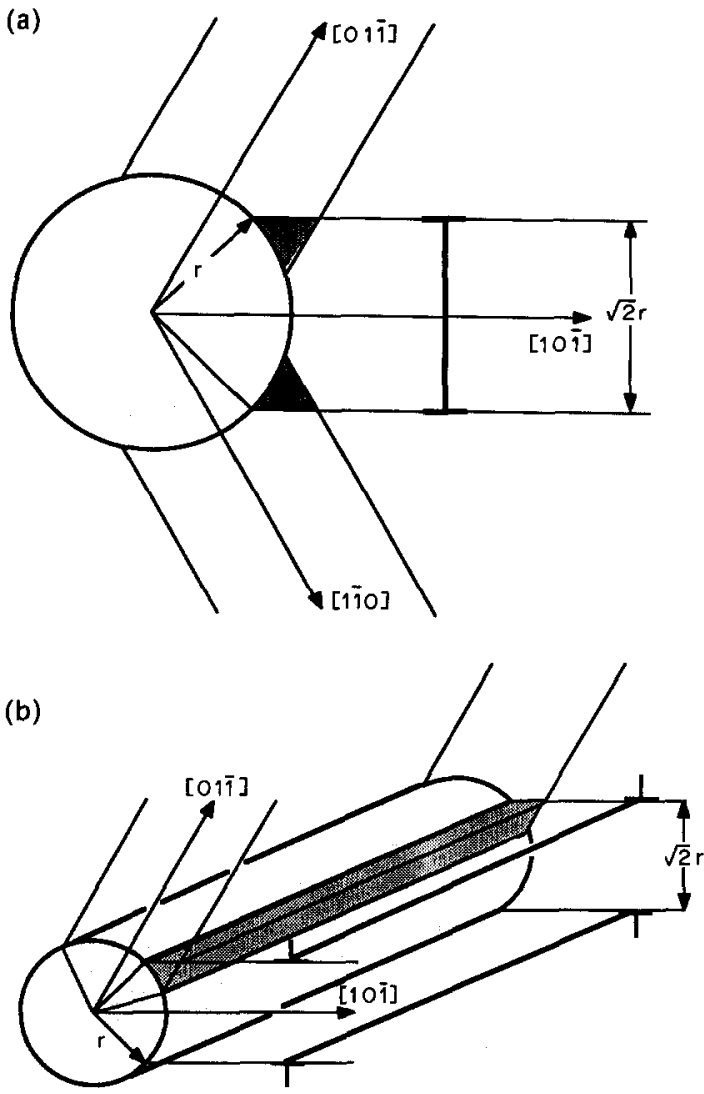

Fig. 9. (a, b) Schematic of prismatic dislocation loops punched in the $\langle 110\rangle$ directions of a f.c.c. lattice from a sphere (a) and a cylinder (b). The shaded volumes represent the intersection of adjacent glide systems.

plastic zone around inclusions, we can then use equation (6) derived above with

$$
\sigma_{\mathrm{y}}=2 \tau_{\mathrm{y}} .
$$

The dislocation density $\rho$ is estimated as the total length of punched prismatic dislocation loops that are needed to relieve the thermal mismatch strains in simple configurations divided by the plastic zone volume. For the sphere, we adopt the model described by Hull and Bacon [46] wherein it is assumed that loops are punched out along all 12 $\langle 110\rangle$ directions on glide cylinders intersecting the sphere along circles where the resolved shear stress is maximum [Fig. 9(a)]; the glide cylinders have then a diameter of $\sqrt{ } 2 r_{\mathrm{s}}$. For the cylinder, it is assumed that the axis of the cylinder is aligned along one of the $\langle 111\rangle$ directions and that infinitely long loops are emitted in the six $\langle 110\rangle$ directions. We assume that, as with the sphere, these loops are emitted where the resolved shear stress is maximum, resulting in a loop width of $\sqrt{ } 2 r_{\mathrm{c}}$ [Fig. 9(b)]. Deviation of the particular fiber orientation from $\langle 111\rangle$ increase the number of loops if no Burgers vectors perpendicular to the fiber axis is available. For this reason, and because a series of loops of same width but shorter length might be generated instead, the present estimate is most likely a lower bound for the actual dislocation density around a cylindrical inclusion.

The number of dislocation loops in the plastic zone around the cylinder $n_{\mathrm{c}}$ and the sphere $n_{\mathrm{s}}$ is now obtained by dividing the volume of the material to be displaced to relieve thermal mismatch by the volume of material removed by each loop

$$
\begin{aligned}
& n_{\mathrm{c}}=\frac{2 \cdot \pi \cdot r_{\mathrm{c}} \cdot \Delta \alpha \cdot \Delta T \cdot r_{\mathrm{c}}}{\sqrt{2} \cdot r_{\mathrm{c}} \cdot b}=\frac{\sqrt{2} \cdot \pi \cdot r_{\mathrm{c}} \cdot \Delta \alpha \cdot \Delta T}{b}, \\
& n_{\mathrm{s}}=\frac{4 \cdot \pi \cdot r_{\mathrm{s}}^{2} \cdot \Delta \alpha \cdot \Delta T \cdot r_{\mathrm{s}}}{\pi \cdot \frac{r_{\mathrm{s}}^{2}}{2} \cdot b}=\frac{8 \cdot r_{\mathrm{s}} \cdot \Delta \alpha \cdot \Delta T}{b}
\end{aligned}
$$

Dividing the total dislocation length by the volume of the plastic zone, the dislocation densities around a cylinder and a sphere are then respectively

$$
\begin{aligned}
& \rho_{\mathrm{c}}=\frac{2 \cdot n_{\mathrm{c}}}{\pi \cdot\left(c_{\mathrm{c}}^{2}-r_{\mathrm{c}}^{2}\right)}=\frac{2 \cdot \sqrt{2} \cdot \Delta \alpha \cdot \Delta T}{b \cdot r_{\mathrm{c}} \cdot\left(x_{\mathrm{c}}-1\right)}, \\
& \rho_{\mathrm{s}}=\frac{3 \cdot n_{\mathrm{s}} \cdot r_{\mathrm{s}}}{2 \cdot \sqrt{2} \cdot\left(c_{\mathrm{s}}^{3}-r_{\mathrm{s}}^{3}\right)}=\frac{6 \cdot \sqrt{2} \cdot \Delta \alpha \cdot \Delta T}{b \cdot r_{\mathrm{s}} \cdot\left(x_{\mathrm{s}}-1\right)}
\end{aligned}
$$

where

$$
\begin{aligned}
& x_{\mathrm{c}}=\left(\frac{c_{\mathrm{c}}}{r_{\mathrm{c}}}\right)^{2}, \\
& x_{\mathrm{s}}=\left(\frac{c_{\mathrm{s}}}{r_{\mathrm{s}}}\right)^{3} .
\end{aligned}
$$

Inserting equations (7), (8) and (10) into equation (6) leads to implicit equations for the radius of the plastic zone in a strain-hardening matrix for the cylinder and the sphere respectively

$$
\begin{aligned}
& \tau_{\mathrm{y} 0}+A \cdot G \cdot \sqrt{\frac{2 \cdot \sqrt{2} \cdot \Delta \alpha \cdot \Delta T \cdot b}{r_{\mathrm{c}} \cdot\left(x_{\mathrm{c}}-1\right)}} \\
& -\frac{2 \cdot \Delta \alpha \cdot \Delta T \cdot E}{(5-4 v) \cdot x_{\mathrm{c}}}=0, \\
& \tau_{\mathrm{y} 0}+A \cdot G \cdot \sqrt{\frac{6 \cdot \sqrt{2} \cdot \Delta \alpha \cdot \Delta T \cdot b}{r_{\mathrm{s}} \cdot\left(x_{\mathrm{s}}-1\right)}} \\
& -\frac{\Delta \alpha \cdot \Delta T \cdot E}{2 \cdot(1-v) \cdot x_{s}}=0 .
\end{aligned}
$$

After some algebraic manipulations, these two equations can be transformed into cubic equations in $x_{\mathrm{c}}$ and $x_{\mathrm{s}}$ respectively as a function of $\Delta \alpha, \Delta T, G, E$, $v, \tau_{\mathrm{y} 0}, b, A$ and $r_{\mathrm{c}}$ or $r_{\mathrm{s}}$ respectively. For the particular cases explored numerically here $\left(\mathrm{AgCl} / \mathrm{Al}_{2} \mathrm{O}_{3}\right.$, $\mathrm{AgCl} /$ glass and $\mathrm{Al} / \mathrm{SiC}$ ), these equations generally yield three roots, two of which are extraneous. With the strain-hardening constant $A$ equal to zero-corresponding to a non strain-hardening matrix - the cubic equation yields an extraneous root $x=1$ and a double root equal to the value found using equation (6) with $\sigma_{y}=2 \tau_{y 0}$ (reference value). With $A>0$ (i.e. for a strain-hardening matrix), the smallest root is still equal to 1 and is discarded since it is nonphysical. Of the other two roots, one is larger than the 


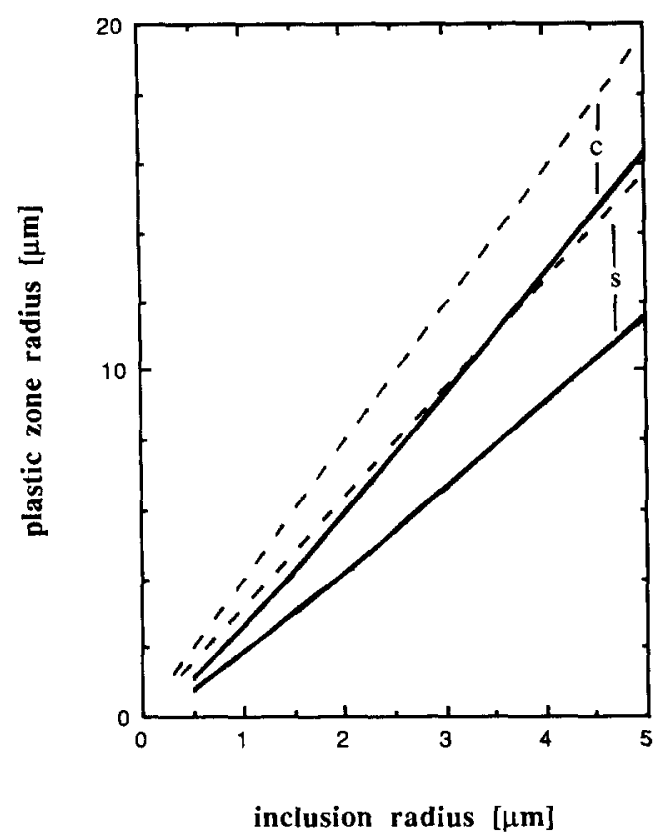

Fig. 10. Plastic zone radius around a SiC cylinder (c) and sphere $(s)$ in a $99.5 \%$ aluminium matrix after a temperature change of $200 \mathrm{~K}$. Materials constants are listed in Table 1. Full curves: strain-hardening matrix [equation (12)]. Dotted curves: non strain-hardening matrix [equation (6)].

reference value and hence physically absurd, the other is smaller than the reference value and therefore retained.

The critical volume fraction $v^{*}$ of inclusions at which their plastic zone are touching is then for the cylinders and spheres respectively

$$
\begin{aligned}
& v_{\mathrm{c}}^{*}=\frac{P_{\mathrm{c}}}{x_{\mathrm{c}}}, \\
& v_{\mathrm{s}}^{*}=\frac{P_{\mathrm{s}}}{x_{\mathrm{s}}},
\end{aligned}
$$

where $P_{\mathrm{c}}$ and $P_{\mathrm{s}}$ are the packing ratios for cylinders and spheres respectively. Since equation (1) was developed for a single inclusion in an infinite matrix, the volume fractions derived above are larger than the upper limit for the range of validity of equation (12).

Results from this model for $\mathrm{SiC}$ spheres and cylinders of radius $1.5 \mu \mathrm{m}$ in a $99.5 \%$ pure aluminium matrix for an arbitrary value for $\Delta T$ of $200 \mathrm{~K}$ are given in Fig. 10, using CTE value for $\mathrm{SiC}$ of $3.4 \cdot 10^{-6} \mathrm{~K}^{-1}$ and constants given in Table 1 . The
Table 1. Thermomechanical parameters of matrix materials (high purity silver chloride and $99.5 \%$ aluminium)

\begin{tabular}{lccc}
\hline Parameter & Unit & $\mathrm{AgCl}$ & \multicolumn{1}{c}{$\mathrm{Al}$} \\
\hline $\mathrm{CTE}$ & $\left(\mathrm{K}^{-1}\right)$ & $3 \cdot 10^{-5}$ & $2.32 \cdot 10^{-5}$ \\
$E$ & $(\mathrm{GPa})$ & 26 & 62 \\
$G$ & $(\mathrm{GPa})$ & 6.9 & 23 \\
$v$ & $(-)$ & 0.343 & 0.33 \\
$b$ & $(\mathrm{~nm})$ & 0.384 & 0.286 \\
$\tau_{\mathrm{y} 0}$ & $(\mathrm{MPa})$ & 0.5 & 8.8 \\
$A$ & $(-)$ & & 0.625 \\
Reference & & {$[67,71]$} & {$[43,72]$} \\
\hline
\end{tabular}

choice of slightly alloyed aluminium for this example rather than the pure metal was dictated by the fact that, even for the thermal strain rates achievable by quenching, pure aluminium deforms by slip only at temperatures below $300 \mathrm{~K}$. With the latter metal, the effect of work hardening on plastic zone radius is even more pronounced for the same value of $\Delta T$ (in a quench from ambient to liquid nitrogen temperatures for example) due to its lower value of $\tau_{y 0}$.

The plastic zone radius that results from use of equation (6) with $\sigma_{\mathrm{y}}=2 \tau_{\mathrm{y}}$ for a non strain-hardening matrix is given in the same figure. The critical volume fraction and dislocation density are given in Table 2 for cylinders and spheres under the same conditions as above. Also listed in Table 2 is the increase of yield stress in shear due to the dislocations present in the matrix [equation (7)]. Due to strain-hardening, the value of the yield stress in the plastic zone around cylinders and spheres is respectively over 1.5 and 2 times the intrinsic flow stress measured by Hansen [43].

\section{DISCUSSION}

A large body of literature exists on dislocation nucleation and geometry around submicroscopic particles in deformed metals, e.g. [33, 45, 47-51]. While very detailed TEM observations and mechanical test results have shed considerable light on the dislocation mechanisms operative in such systems, these results are not directly applicable to the present problem because the volume of the second phase in the present study is typically more than three orders of magnitude larger. The completion in an unimpeded manner of such processes as double cross-slip [48] are much less probable with large inclusions because the length of the involved dislocations is much larger. The probability of interaction before completion of the process is therefore much higher and the activation energy is increased by an order of magnitude.

Table 2. Packing ratio, critical volume ratio [equation (13)], dislocation density [equation

(10)] and increase of yield stress in shear [equation (7)] for different arrays of silicon

carbide spheres and cylinders of radius $1.5 \mu \mathrm{m}$ in a strain-hardening $99.5 \%$ aluminium

\begin{tabular}{|c|c|c|c|c|c|}
\hline & Array & $P$ & $v^{*}(\%)$ & $\rho\left(\mathrm{m}^{-2}\right)$ & $A G b \sqrt{ } \rho(\mathrm{Pa})$ \\
\hline \multirow[t]{3}{*}{ Sphere } & f.c.c. & $\sqrt{2 \pi / 6}$ & 6.1 & $5.9 \cdot 10^{12}$ & $10 \cdot 10^{6}$ \\
\hline & b.c.c. & $\sqrt{3 \pi / 8}$ & 5.6 & $5.9 \cdot 10^{12}$ & $10 \cdot 10^{6}$ \\
\hline & Cubic & $\pi / 6$ & 4.3 & $5.9 \cdot 10^{12}$ & $10 \cdot 10^{6}$ \\
\hline \multirow[t]{2}{*}{ Cylinder } & Hexagonal & $\sqrt{3} \pi / 6$ & 5.1 & $1.7 \cdot 10^{12}$ & $5.4 \cdot 10^{6}$ \\
\hline & Square & $\pi / 4$ & 4.4 & $1.7 \cdot 10^{12}$ & $5.4 \cdot 10^{6}$ \\
\hline
\end{tabular}
matrix submitted to a change of temperature $\Delta T=200 \mathrm{~K}$ 


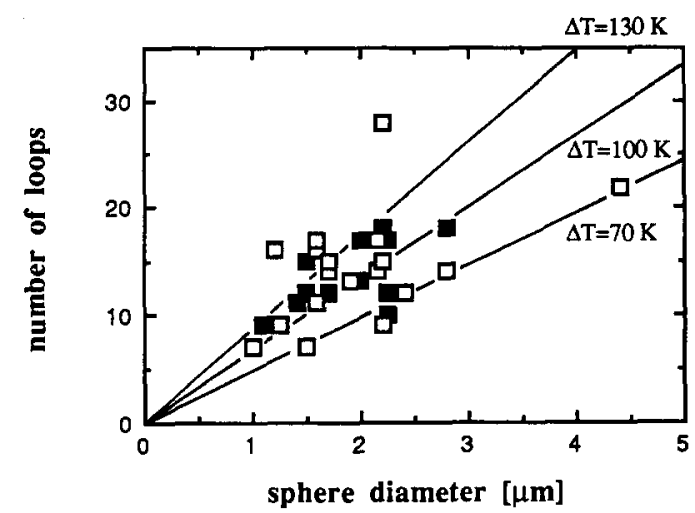

Fig. 11. Total number of prismatic dislocations loops punched by glass spheres in $\mathrm{AgCl}$ as a function of the sphere diameter. Open squares: single row of loops. Solid squares: double rows of loops. Lines: calculations from equation (18) for different values of $\Delta T$.

Another important difference is that most of the earlier studies investigated particles submitted to shear whereas in the present study the strains are purely dilatational. Finally, the interface in the present case is incoherent, while many earlier investigations studied systems with coherent interfaces; this has a large effect on the energy considerations involved in such processes as prismatic punching [33].

While not directly transposable to the problem treated in this study, these previous investigations indicate however that the total dislocation density we assumed to be generated at the reinforcement is most likely a rough approximation. This relative crudeness is justified by the absence of detailed information on the specifics of dislocation emission in the tangled regions by large incoherent particles and the simplicity of the resulting expressions [equation (12)] for the plastic zone radius.

In what follows, we discuss the geometry of dislocations forming the plastic zone around microspheres and fibers. We then present experimental evidence of strain-hardening in the plastic zone surrounding the fibers and derive the value of $\Delta T$ from the number of loops punched by microspheres. We finally compare the predictions of the simple model presented above with the experimentally determined radius of the plastic zone around fibers and spheres.

\subsection{Dislocation around spherical inclusions}

Plastic zones of irregular size, an example of which is given in Fig. 4, present a mode of strain relief intermediate between prismatic loop trains punched along crystallographic directions and an isotropic spherical shell of plastically deformed matrix. It seems that prismatic loops were punched out along crystallographic directions but became thereafter entangled. TEM investigations to date have generated similar observations on particles over an order of magnitude smaller [47, 52]. This suggests that the spherical plastic shells observed around more than half of the embedded particles (Fig. 2)-similar to spherical zones of tangled dislocations around submicroscopic particles reported by Kinsman et al. [53]-resulted from punched loops which were entangled early in the punching process. This lends some credence to our estimation of the final dislocation density in the regular spherical plastic zones modelled above.

When more than one row of loops were found, they were very often at opposite sides of the spheres. No glass sphere was found to have punched loops on two adjacent glide cylinders without the presence of a zone of tangled dislocations between the punched loops and the particle. One of the rows was almost invariably much shorter than the other. This could be due to interactions between loops in the intersection of the glide cylinders close to the sphere [shaded area in Fig. 9(a)]. As pointed out by Johnson and Lee [54], it is energetically possible for two loops on adjacent cylinders in an f.c.c. crystal to interact and form a sessile product dislocation. This is more likely to happen the larger the particle, since the intersection volume between glide cylinders increases in size. Thus, for the large spheres investigated here, plastic zones of tangled dislocations form whenever two adjacent glide cylinders are active. We therefore expect plastic deformation of the matrix around inclusions in the form of a shell of tangled dislocations to be more prevalent over rows of prismatic loops in MMCs compared to dispersion hardened metals because inclusion dimensions are much larger in the former materials.

The intermediate case illustrated in Fig. 3 could be due to a first stage of relaxation where the active glide cylinders were not adjacent, followed by the activation of additional glide cylinders leading to tangles. Similar TEM micrographs on submicroscopic particles have been published by Makenas and Birnbaum [55].

Figure 11 shows the total number of punched prismatic loops as a function of the sphere diameter, for rows of loops which were not separated from the sphere by any tangled dislocations. There is no systematic difference in the total number of loops emitted by spheres with one or two rows of loops, i.e. the number of loops in single rows is on the average twice that for double rows for a given sphere diameter. This suggests that one row of loops can relax the sphere as efficiently as two on each side and in turn implies that short range diffusion or glide occurs in the vicinity of the interface. The observation that with these spheres trains of loops were emitted only along one direction is an indication that nucleation of loops in other directions is perturbed by emission of the first row of dislocations. However, with precipitates of silver in the matrix much smaller than the glass spheres, we observed dislocation punched along most or all crystallographic directions, similar to what has been reported by various investigators in silver chloride $[23,56]$ and metals [57-60]. This indicates that the mechanisms for relief of thermal 


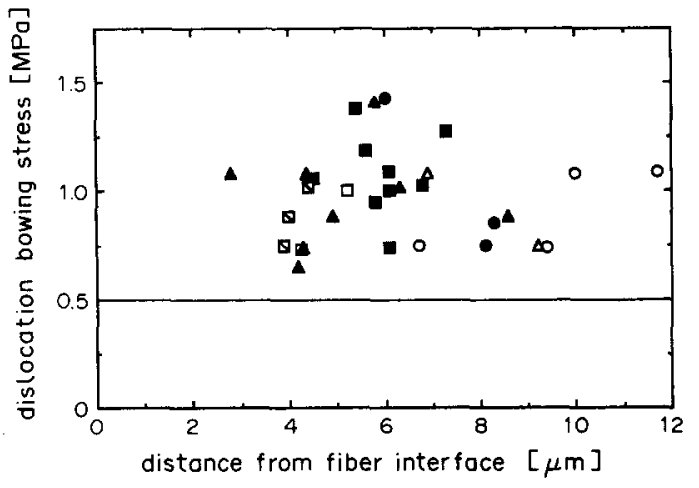

Fig. 12. Stress of pinned dislocations as calculated from equation (12) in the plastic zone surrounding alumina fibers in an $\mathrm{AgCl}$ matrix (see Fig. 5) as a function of radial distance from the interface. The various symbols represent different fibers. The lower horizontal line is the value of the microyield stress in shear as measured by other investigators $[67-68]$.

mismatch depend on inclusion size. Extrapolation to MMCs of data gathered with dispersion hardened metals will therefore not always be valid.

\subsection{Dislocations around cylindrical inclusions}

Childs and Slifkin [16] published a micrograph of a $200 \mu \mathrm{m}$ long dislocation in silver chloride pinned at many places and bowed due to an external stress. Our observations of pinned, bowed dislocations close to fibers are similar. The same authors have also published two micrographs of punched trains of dislocation loops at elongated inclusions in silver chloride. In both their and our work, trains of prismatic dislocation loops were found to emanate from the fiber ends. One of their micrographs features loops of dislocations punched along the inclusion length as well, which we only observed in very few instances. Relaxation of radial mismatch strains was found in all other fibers to result in the formation of a cylindrical plastic zone containing tangled dislocations. This observation is to be expected in view of the fact that unless the fiber axis is perfectly perpendicular to the glide directions of emitted prismatic loops, significant interference will result between emitted dislocations of differing Burgers vector. Even when the fiber is optimally oriented [as in Fig. 9(b)], loops on overlapping glide directions will be able to form sessile products as in the case of spherical inclusions. One would therefore expect that the total emitted dislocation length in the plastic zone surrounding the fibers is estimated with reasonable accuracy as that resulting from elongated punched prismatic loops [equation (9a)] since the mechanism of emission is likely to be similar, and since dislocations are of a length larger than the fiber diameter, as exemplified in Fig. 5.

With short fibers, the peanut-shaped plastic zones we observed (Fig. 7) are similar to those predicted by Dutta et al. [31] using finite element analysis for an elastic, perfectly plastic matrix. These confirm that the dark unresolved regions found surrounding the glass spheres and the alumina fibers are indeed zones of plastically deformed matrix.

\subsection{Flow stress of the dislocations}

Mitchell [56] decorated dislocations in silver chloride under load which were formed by an indenter and also observed bowed dislocations pinned at their ends, as did Childs and Slifkin [16]. Careful examination of long dislocations in the plastic zone surrounding fibers in this investigation reveals that, in several areas, the dislocations were similarly pinned and bowed due to the local stress field (Fig. 5). Since they were in the vast majority of cases found to be bowed away from the fiber on both of its sides, the stress to which they were subjected can be assumed to emanate from the fiber, rather than from some unknown extraneous source. This local elastic stress is unable to move these particular dislocations any further and is therefore lower than the local yield stress in shear of the matrix. Brown and Stobbs [45] also observed around subimicroscopic silica particles in deformed copper that the dislocations in the plastic zone had high curvature compatible with an increased local flow stress.

Assuming that the pinned dislocations formed a segment of a circle, their radius of curvature $R$ was calculated by measuring on enlarged micrographs the distance between the anchor points $L$ as well as the maximum deflection $H$ from the line running through the two anchor points, using the relationship [61]

$$
R=\frac{L^{2}}{8 \cdot H}+\frac{H}{2}
$$

from which it is possible to calculate the local stress $\sigma[62]$

$$
\begin{aligned}
& \sigma=\frac{G \cdot b}{4 \cdot \pi \cdot R \cdot(1-v)} \\
& \times\left\{\frac{v}{2}-1+\left[1-\frac{v}{2} \cdot\left(3-4 \cos ^{2} \beta\right)\right] \cdot \ln \left(\frac{L}{R_{0}}\right)\right\}
\end{aligned}
$$

where $G$ is the matrix shear modulus, $v$ its Poisson's ratio, $R_{0}$ the core distance of the dislocation and $\beta$ the angle between the dislocation line and the Burgers vector.

The angle $\beta$ is unknown and the square of its cosine is taken as 0.5 (the average of $\cos ^{2} \beta$ ). The core distance is taken as $33 \%$ the value of the Burgers vector, as determined by Puls and So [63] for sodium chloride. These two parameters have little influence on the numerical result. The Burgers vector was assumed to be along $\{110\}$, the main slip direction in silver chloride [12]. The Poisson's ratio was calculated as 0.343 from an average of the values of elastic constants determined by three investigators [64-66]; other materials data are listed in Table 1. The stresses for pinned bowed dislocations such as the ones visible 


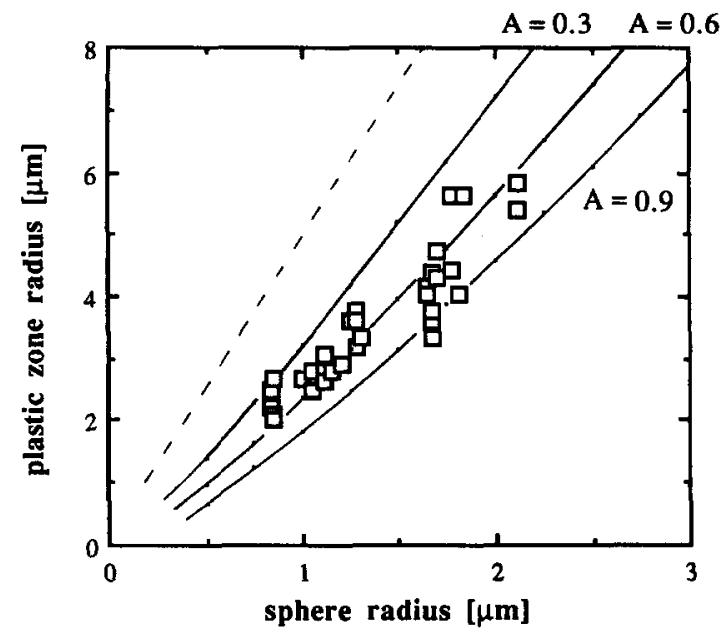

Fig. 13. Radius of plastic zone surrounding a sphere in an $\mathrm{AgCl}$ matrix as a function of the radius of the corresponding sphere. Squares: experimental data. Solid curves: strainhardening matrix with different values of [equation (12b)]. Dashed curve: nonstrain-hardening matrix [equation (6b)]

in Fig. 5 are plotted in Fig. 12. It is found that the local stress within the plastic region is independent of the distance from the fiber and varies between $0.6 \mathrm{MPa}$ and $1.45 \mathrm{MPa}$. The lower bound is in good agreement with a value of $0.5 \mathrm{MPa}$ for the microyield shear stress of pure silver chloride measured at room temperature by other investigators $[67,68]$. Haasen and Skrotsky [68] also found that the presence of 300 and $500 \mathrm{ppm}$ of calcium chloride in the matrix hardly changed the value of the critical resolved shear stress. This indicates that the $500 \mathrm{ppm}$ of cuprous chloride present in the sample used in this investigation should have very little effect on the yield stress of silver chloride, especially since the monovalent cuprous ions are expected to interfere less with dislocations than the divalent calcium ions investigated by Haasen and Skrotsky [68]. In a matrix showing no strainhardening, one would expect the stress in the plastic zone to be constant and equal to the microyield stress, i.e. around 0.5 MPa. The data shown in Fig. 12 thus indicate that the matrix is strain hardened, as found by many authors with silver chloride in tension ([34-36, 68-69]).

\subsection{Determination of $\Delta T$}

Each punched-out prismatic loop of Burgers vector $b$ emitted by a sphere of diameter $d_{\mathrm{s}}$ relaxes the thermal mismatch in the direction of emission of the loops by a strain $\epsilon_{1}$ approximated by

$$
\epsilon_{1}=\frac{b}{d_{\mathrm{s}}}
$$

assuming that the emitted loop has the same diameter as the sphere (as observed experimentally) and neglecting strains in the direction where no loops are emitted. The total mismatch strain $\epsilon_{\mathrm{m}}$ between sphere and matrix is given by equation (2). Assuming that this mismatch is completely relaxed along one direc- tion by punching of coaxial prismatic loops, the total number of loops $n_{\mathrm{s}}$ is equal to

$$
n_{\mathrm{s}}=\frac{\epsilon_{\mathrm{m}}}{\epsilon_{1}}
$$

Inserting equations (2) and (16) into (17) yields

$$
\Delta T=\frac{n_{\mathrm{s}} \cdot b}{d_{\mathrm{s}} \cdot \Delta \alpha} .
$$

The right hand side of equation (18) would be divided by a factor 2 if it were assumed that loops can be punched out along all $12\{110\}$ directions as described by Hull and Bacon [38]. Figure 11 shows the measured number of loops $n_{\mathrm{s}}$ as a function of sphere diameter. The only spheres considered were those that did not exhibit any tangled dislocations between the row of loops and the sphere/matrix interface in the glide cylinder direction, i.e. for cases where relaxation took place solely by prismatic loop punching. The best fit line passing through the origin gives a value of $100 \mathrm{~K}$ for $\Delta T$ using equation [18]; lines corresponding to $\Delta T$ values of 70 and $130 \mathrm{~K}$ are also shown on the same figure. While there is considerable scatter in the data, it can be safely assumed that the value of $\Delta T$ is $100 \pm 30 \mathrm{~K}$. This translates into an homologous temperature $T_{\mathrm{c}} / T_{\mathrm{m}}$ for the onset of slip of $0.55 \pm 0.04$, a result in good agreement with the value of 0.50 for sodium chloride given by Frost and Ashby [70] for the transition from power lawcreep to plasticity at the strain rate corresponding to the rate of mismatch due to the experimental cooling rate of $1 \mathrm{~K} / \mathrm{s}$. Since sodium chloride and silver chloride are in the same isomechanical group, the homologous temperature of transition from one deformation mechanism to another is expected to be the same [70]. The scatter in Fig. 11 can be due to loops which disappeared due to combination with point defects produced during quenching, unresolved loops not counted, superimposed macroscopic stresses, incompletely relieved stresses by the sphere, varying loop diameter, etc. Most of these factors indicate that $\Delta T$ is probably somewhat above $100 \mathrm{~K}$.

\subsection{Comparison of plastic zone around spheres with theory}

For the case of spherical plastic zones around particles, the plastic zone radius $c_{\mathrm{s}}$ is plotted in Fig. 13 as a function of particle radius $r_{\mathrm{s}}$. Only cases where the spheres were isolated from neighbours and where the plastic zone was spherical were considered. As predicted by equation (6b), there is a linear relationship between the radius of the sphere and the radius of the plastic zone. Using values for silver chloride listed in Table 1 and a value of $3 \cdot 10^{-6} \mathrm{~K}^{-1}$ for the CTE of glass, equation (6b) yields an unreasonably low value of $18 \mathrm{~K}$ for $\Delta T$ compared to the previously determined value of $100 \mathrm{~K}$. Alternatively, for $\Delta T=100 \mathrm{~K}$, equation (6b) yields a value of $5.7 \mathrm{MPa}$ for $\sigma_{y}$ which corresponds to the stress value measured in simple tension on silver chloride by Carnahan et al. 
[69] at a strain of $2.1 \cdot 10^{-4}$, which is equivalent to $\epsilon_{\mathrm{m}}$ with $\Delta T=100 \mathrm{~K}$ [equation (2)]. Their measurements were made on annealed samples which all showed significant work hardening (ultimate tensile strength more than ten times higher than the microyield stress of $1 \mathrm{MPa}$ measured by Sprackling [67]), independently of the grain size. Equation (12b), on the other hand, gives very good agreement with the data points for values of $\Delta T=100 \mathrm{~K}$ and $A=0.6$. Equation (12b) is plotted in Fig. 13 for these values as well as for values of $A$ equal to 0.3 and 0.9 . For comparison, equation (6b) is also plotted in the same figure with $\Delta T=100 \mathrm{~K}$ and $\sigma_{\mathrm{y}}=2 \tau_{\mathrm{y} 0}=1 \mathrm{MPa}$.

\subsection{Comparison of plastic zone around fibers with theory}

The radius of the plastic zone around fibers was also measured for 45 fibers of aspect ratio larger than 20 , in cases where the plastic zone was a cylinder with a constant radius. For the fibers used in this investigation which have a radius of $1.5 \mu \mathrm{m}$, the radius of the plastic zone was measured as $10.2 \mu \mathrm{m}$, with a standard deviation of $2.7 \mu \mathrm{m}$. Using equation (12a) with the previously determined values of $\Delta T=100 \mathrm{~K}$ and $A=0.6$, we calculate a plastic zone radius $c_{\mathrm{c}}$ equal to $6.2 \mu \mathrm{m}$. Equations (7) and (10a) predict a value of $2 \mathrm{MPa}$ for the value of the yield stress in shear within the plastic zone. As expected, all measured stress values are below this value (Fig. 12). Changing the parameters $\Delta T$ and $A$ by a factor $30 \%$ (i.e. $130 \mathrm{~K}$ and 0.42 respectively), yields a value of $7.6 \mu \mathrm{m}$ for $c_{\mathrm{c}}$, within the interval of confidence of measured values. The yield stress in shear of the strain hardened matrix becomes $1.4 \mathrm{MPa}$, equal to the maximum value measured experimentally (Fig. 12), as should be the case. The discrepancy of $30 \%$ with the values determined for the spheres can be explained by the fact that equation (12a) does not take into account the contribution of the axial mismatch to the radial plastic zone, since the model was developed for an infinitely long fiber. An alternate explanation can be found in the observation that subgrain boundaries were generally found to exist at the interface separating the plastic zone from the unstrained matrix around fibers. A smaller plastic zone size should result due to the locally enhanced dislocation density at these subgrain boundaries within the plastic zone. Equation (6a) yields a value of $17.4 \mu \mathrm{m}$ for $c_{\mathrm{c}}$, far in excess of the range of measured values.

From the above results, it appears that equation (12) which takes into account strain-hardening of the matrix fit well the experimental data for both spherical and cylindrical inclusions, while equation (6) which assumes a perfectly plastic matrix fails to do so. We conclude that strain-hardening must be taken into account in modelling matrix plastic deformation around reinforcements in MMCs, and that with reinforcement volume fractions below the values given by equation (13), equation (12) should yield an adequate estimation of plastic zone size in a coarsegrained, single-phase matrix. As shown in Fig. 10, dislocations punched by the reinforcing phase should have a significant effect on the size of the plastic zone in pure aluminium. It must however be kept in mind that this effect will become less pronounced with larger values of $\tau_{y 0}$ typical of alloys. Furthermore, the simple calculation presented above loses its validity when other obstacles (grain and subgrain boundaries, second phases and other reinforcements) also impede dislocation motion in the vicinity of the reinforcement.

\section{CONCLUSIONS}

Silver chloride containing short alumina fibers $3 \mu \mathrm{m}$ in diameter or glass microspheres of diameter ranging from 1 to $5 \mu \mathrm{m}$ is used as a model material for the study of thermally induced dislocations in metal matrix composites. The dislocations are decorated in the bulk matrix at room temperature and observed by transmission optical microsopy. Despite its lower resolution, this technique complements TEM investigations of MMCs because of the large volume of undisturbed material that can be investigated.

Rows of prismatic loops punched out by glass spheres without subsequent entanglement at the sphere/matrix interface are found only along one direction. The number of loops emitted by glass spheres is found to be proportional to the sphere diameter, but independent of whether the loops are emitted in one or two rows. By counting the emitted loops, it is concluded that relaxation by slip occurs within a temperature interval of $100 \pm 30 \mathrm{~K}$, smaller that the total temperature difference experienced during quenching from annealing temperatures but equal to an homologuous transition temperature from creep to slip of 0.55 , in agreement with Frost and Ashby [70].

The mechanism of prismatic loop punching at the end of a fiber is confirmed and rows of prismatic loops at equilibrium extending at large distances from fibers in the matrix are observed.

Plastic zones containing tangled dislocations generally result along the sides of the fibers. The stress within this plastic zone is measured using the curvature of pinned dislocations and found to be up to thrice the resolved yield stress in shear of the matrix. This observation leads to the conclusion that the matrix is locally strain hardened. The measured dislocation stress is also lower than the yield stress in shear calculated from the dislocation density in the plastic zone, as it should be.

Plastic zones formed by tangles of dislocations are observed around spheres and fibers. A simple calculation of the radii of these plastic zones taking into account strain-hardening of the matrix is presented and confirmed by experimental data. 
Acknowledgements-This work was supported by the Innovative Science and Technology Program of the S.D.I.O. through the Office of Naval Research, Contract No. N00014-85-K-0645, monitored by Dr S. G. Fishman at O.N.R. and Dr J. A. Cornie at M.I.T. We are grateful for a graduate fellowship granted to D.C.D. by International Business Machines Corporation. We also wish to express our most grateful apprecation to Professor K. C. Russell at M.I.T. for stimulating discussions and to Professor J. W. Mitchell for advice in the initial stages of our experimental research.

\section{REFERENCES}

1. K. K. Chawla and M. Metzger, J. Mater. Sci. 7, 34 (1972).

2. K. K. Chawla and M. Metzger, Metall. Trans. 8A, 1680 (1977).

3. Y. Flom and R. J. Arsenault, Mater. Sci. Engng 75, 151 (1985).

4. S. P. Rawal, L. F. Allard and M. S. Misra, Interfaces in Metal Matrix Composites (edited by A. K. Dhingra and S. G. Fishman), p. 211. A.I.M.E., New York (1986).

5. W. Funk and E. Blank, Metall. Trans. 19A, 987 (1988).

6. Q. Li, J. Megusar, L. J. Masur and J. A. Cornie, Mater. Sci. Engng. A117, 199 (1989).

7. S. R. Nutt and J. M. Duva, Scripta metall. 20, 1055 (1986).

8. M. Taya and T. Mori, Acta metall. 35, 155 (1987).

9. M. Vogelsang, R. J. Arsenault and R. M. Fisher, Metall. Trans. 17A, 379 (1986).

10. S. P. Rawal, L. F. Allard and M. S. Misra, Proc. Sixth Int. Conf. on Composite Materials ICCM6 (edited by F. L. Matthews, N. C. R. Buskell and J. M. Hodginson), p. 2.169. Elsevier Applied Science, Amsterdam (1987).

11. A. W. Stepanov, Phys. Z. Sowjet. 8, 25 (1935).

12. M. T. Sprackling, The Plastic Deformation of Simple Ionic Crystals. Academic Press, New York (1976)

13. J. M. Hedges and J. W. Mitchell, Phil. Mag. 7, 223 (1953).

14. J. M. Hedges and J. W. Mitchell, Phil. Mag. 7, 357 (1953).

15. H. Layer, M. G. Miller and L. M. Slifkin, J. appl. Phys. 33, 478 (1962).

16. L. M. Childs and C. B. Slifkin, Br. J. appl. Phys. 16, 771 (1965).

17. J. R. Haynes and W. Shockley, Phys. Rev. 82, 935 (1951).

18. P. Süptitz, Z. Phys. 153, 174 (1958).

19. P. V. Clark and J. W. Mitchell, J. Phot. Sci. 4, 1 (1956).

20. J. W. Mitchell, Dislocations and Mechanical Properties of Crystals, p. 69. Wiley, New York (1957).

21. D. A. Jones and J. W. Mitchell, Phil. Mag. 3, 1 (1958).

22. J. W. Mitchell, Faraday Soc. Disc. 28, 242 (1959).

23. A. S. Parasnis and J. W. Mitchell, Phil. Mag. 8, 171 (1959).

24. C. A. Hoffman, J. Engng. Mater. Tech. 95, 55 (1973).

25. G. Garmong, Metall. Trans. 5, 2183 (1974).

26. J. K. Lee, Y. Y. Earmme, H. I. Aaronson and K. C. Russell, Metall. Trans. 11A, 1837 (1980).

27. Y. Y. Earmme, W. C. Johnson and J. K. Lee, Metall. Trans. 12A, 1521 (1981).

28. A. R. T. de Silva and G. A. Chadwick, J. Mech. Phys. Solids 17, 387 (1969).

29. M. Vedula, R. N. Pangborn and R. A. Queeney, Composites 19, 55 (1988).

30. M. Vedula, R. N. Pangborn and R. A. Queeney, Composites 19, 133 (1988).

31. I. Dutta, D. L. Bourell and D. Latimer, J. comp. Mater. 22, 829 (1988)
32. R. Hill, The Mathematical Theory of Plasticity. Oxford Univ. Press (1950).

33. L. M. Brown and G. R. Woolhouse, Phil. Mag. 21, 329 (1970).

34. A. W. Stepanow, Phys. Z. Sowjet. 6, 312 (1934).

35. A. W. Stepanow, Phys. Z. Sowjet. 8, 25 (1935).

36. B. M. Axilrod and J. J. Lamb, J. appl. Phys. 19, 213 (1948).

37. G. I. Taylor, Proc. R. Soc. 145A, 362 (1934).

38. J. E. Bailey and P. B. Hirsch, Phil. Mag. 5, 485 (1960).

39. A. S. Keh, Direct Observation of Imperfections in Crystals, p. 213. Interscience, New York (1962).

40. J. D. Livingston, Acta metall. 10, 229 (1962).

41. J. A. Venables, Phil. Mag. 7, 1969 (1962).

42. S. Mader, A. Seeger and H. Thieringer, J. appl. Phys. 34, 3376 (1963)

43. N. Hansen, Acta metall. 25, 863 (1977)

44. W. in der Schmitten and P. Haasen, J. appl. Phys. 32, 1790 (1961).

45. L. M. Brown and W. M. Stobbs, Phil. Mag. 23, 1201 (1971).

46. D. Hull and D. J. Bacon, Introduction to Dislocations, 3rd edn, p. 166. Pergamon Press, Oxford (1984).

47. M. F. Ashby, S. H. Gelles and L. E. Tanner, Phil. Mag. 19, 757 (1969).

48. P. B. Hirsch and F. J. Humphreys, Proc. R. Soc. 318A, $45(1970)$

49. F. J. Humphreys and P. B. Hirsch, Proc. R. Soc. 318A, 73 (1970)

50. D. Gould, P. B. Hirsch and F. J. Humphreys, Phil. Mag. 30, 1353 (1974)

51. F. J. Humphreys and P. B. Hirsch, Phil. Mag. 34, 373 (1976).

52. M. H. Lewis and J. M. Martin, Acta metall. 11, 1207 (1963).

53. K. R. Kinsman, J. W. Sprys and R. J. Asaro, Acta metall. 23, 1431 (1975).

54. W. C. Johnson and J. K. Lee, Acta metall. 31, 1033 (1983).

55. B. J. Makenas and H. K. Birnbaum, Acta metall. 28, 979 (1980).

56. J. W. Mitchell, Growth and Perfection of Crystals, p. 386. Wiley, New York (1958)

57. A. Lawley and H. L. Gaigher, Phil. Mag. 8, 1713 (1963).

58. A. Eikum and G. Thomas, Acta metall. 12, 537 (1964).

59. U. Bertocci, C. Bertocci and F. W. Young, J. appl. Phys. 40, 1674 (1969).

60. M. Shimotomai and R. R. Hasiguti, Japan Inst. Met. J. 43, 292 (1979)

61. W. H. Beyer, Standard Mathematical Tables, 25th edn, p. 143. CRC Press, Baton Rouge, La (1979).

62. J. P. Hirth and J. Lothe, Theory of Dislocations, 2nd edn, p. 752. Wiley-Interscience, New York (1982).

63. M. P. Puls and C. B. So, Physica status solidi 98A, 87 (1980).

64. A. V. Stepanov and I. M. Eidus, Zh. Eksperim. Teor. Fiz. 29, 669 (1955)

65. J. Vallin, Arkiv. Fysik. 34, 367 (1967)

66. W. Hidshaw, J. T. Lewis and C. V. Briscoe, Phys. Rev. 2, 876 (1967).

67. M. T. Sprackling, Phil. Mag. 13, 1293 (1966)

68. P. Haasen and W. Skrotski, J. Physique 42, C3-119 (1981).

69. R. D. Carnahan, T. L. Johnston, R. J. Stokes and C. H. Li, Trans. metall. Soc. A.I.M.E. 221, 45 (1961).

70. H. J. Frost and M. F. Ashby, Deformation-Mechanism Maps. Pergamon Press, New York (1982).

71. Gmelins Handbuch der anorganischen Chemie: Silber B1 (edited by Gmelins Institut), Verlag Chemie (1971).

72. Metals Handbook, Desk Edition (edited by H. E. Boyer and T. L. Gall). Am. Soc. Metals, Metals Park, Ohio (1985). 


\section{APPENDIX}

$a=$ equivalent hole radius

$A=$ strain-hardening constant

$b=$ matrix Burgers vector

$c=$ plastic zone radius

$d=$ inclusion diameter

$E=$ matrix elastic modulus

$G=$ matrix shear modulus

$H=$ maximum deflection of a bowed dislocation from the line running between its anchor points

$L=$ distance between the anchor points of a bowed dislocation

$n=$ dislocation number around an inclusion

$P=$ packing ratio

$r=$ inclusion radius

$R=$ radius of curvature of a bowed dislocation

$R_{0}=$ dislocation core distance

$T_{\mathrm{a}}=$ annealing temperature

$T_{\mathrm{c}}=$ creep/glide transition temperature of the matrix

$T_{\mathrm{m}}=$ melting temperature of the matrix
$T_{0}=$ observation temperature

$v^{*}=$ critical volume fraction of inclusions

$x=$ dimensionless plastic zone parameter

$\beta=$ angle between dislocation line and its Burgers vector

$\Delta \alpha=C T E$ difference between matrix and inclusion

$\Delta r=$ matrix displacement at the interface

$\Delta T=$ temperature interval where slip is operative

$\epsilon_{1}=$ strain relieved by a prismatic loop

$\epsilon_{\mathfrak{m}}=$ matrix stain due to inclusion

$v=$ matrix Poisson's ratio

$\rho=$ matrix dislocation density

$\sigma=$ stress

$\sigma_{\mathrm{y}}=$ matrix tensile yield stress

$\tau_{y}=$ matrix shear flow stress

$\tau_{y 0}=$ matrix intrinsic shear flow stress

Subscrip

$\mathrm{c}=$ cylinder or fiber

$\mathrm{s}=$ sphere 